\title{
IMMUNE RESPONSE IN MALIGNANT GLIOMA
}

\author{
I. Koev ${ }^{1}$, E. Slavov ${ }^{2}$, D. Staykov ${ }^{1}$, K. Halacheva ${ }^{2}$, V. Sarafian ${ }^{1}$ \\ 1) Clinic of Neurosurgery; Department of General and Clinical Pathology, \\ Department of Biology, Medical University - Plovdiv, Bulgaria; 2) Department of \\ Molecular Biology, Medical Genetics and Immunology, Trakia University, Stara \\ Zagora, Bulgaria
}

\section{SUMMARY}

Objective: Malignant gliomas are primary brain tumors with excessive mortality and high resistance to chemotherapy and radiotherapy. The survival time for glioblastoma multiforme is about 6-12 months. As key pathogenetic mechanisms are recognized the massive necrosis, angiogenesis and hypoxia within the tumor, as well as the resistance to apoptosis. It is also suspected that altered immune response might contribute to the fatal clinical outcome.

The aim of the present study was to determine the immune status of patients with malignant gliomas.

Material and methods: Peripheral blood lymphocytes were collected preoperatively from 9 patients (aged 57-76) diagnosed as anaplastic astrocytoma grade III $(n=4)$ and glioblastoma multiforme $(n=5)$. The following lymphocyte populations were analyzed by flow cytometry: CD19+, CD3+, $\mathrm{CD} 3+\mathrm{CD} 4+, \mathrm{CD} 3+\mathrm{CD} 8+, \mathrm{CD} 3-\mathrm{CD} 56+, \mathrm{CD} 3+\mathrm{CD} 56+$, CD3+CD25+, CD8-CD11b+, CD8+CD11b+, CD8+CD11b-. The results obtained were compared to reference values for each cell population.

Results: No significant alterations were detected in $\mathrm{CD} 19+, \mathrm{CD} 3+, \mathrm{CD} 3+\mathrm{CD} 4+, \mathrm{CD} 3+\mathrm{CD} 8+$ cells, but the CD4/CD8 ratio was below the reference range in some cases. No obvious decrease in (CD3-CD56+) NK cells and (CD3-CD56+) NKT cells was observed in most patients. A reproducible phenomenon of increased CD8+CD11b+ and decreased CD8+CD11b- cells was noticed.

These preliminary results suggest that the immune response in patients with malignant glioma is seriously disregulated. The rapid clinical deterioration, relapses and high mortality could be at least partially explained with the suppressed activity of NK-cells which are the major cytotoxic antitumoral cells. The increase in the population of activated suppressor-effector cells also contributes to the unfavourable outcome in malignant brain tumors.

Conclusion: This pilot study reveals the presence of altered immune response in malignant gliomas and opens possibilities for prospective investigations concerning immune status and clinical outcome.

Key words: glioma, immune response, immune suppression

\section{INTRODUCTION}

Malignant glial brain tumors and especially glioblastoma multiforme (GBM) are highly aggressive primary tumors. GBM is refractory to traditional treatment such as surgery, radiation and chemotherapy. Genetic abnormalities are associated with aberrant activation of inhibition of cellular transduction pathways and resistance to therapy. Multitargeted kinase inhibitors or combinations of agents affecting various mitogenic mechanisms are discussed as new treatment strategies (1).

The destructive nature of gliomas is also strongly interconnected with their ability to disturb the anti-tumoral immune response. Despite of the active research in this filed little progress in the treatment of GBM has been made so far. At least partially the lack of advance is due to the search of how glial tumor cells are capable of both modulating host immune response and neutralizing immune-based therapies (2).

Different mechanisms are suspected to contribute to systemic immune suppression in glioblastoma. The interplay between altered monocyte phenotypes and tumor factors (3) and the role of interleukin-12 and NK-cells $(4,5)$ are regarded as key factors in this process. Recently, it was proven that glioblastoma cancer-initiating cells inhibited T-cell proliferation and activation, induced regulatory $\mathrm{T}$ cells, and triggered T-cell apoptosis (6). Although the central nervous system is conventionally regarded as "immunologically privileged" current discoveries suggest that the immune system plays an important role in malignant brain tumors.

The still undefined mechanisms of immunoresistance in gliomas and the lack of immunological investigations of glioma patients in Bulgaria motivated the present study. Its aim is to determine the immune status of patients with malignant gliomas.

\section{MATERIALAND METHODS}

Nine patients (aged 57-76) with anaplastic astrocytoma grade III $(n=4)$ and glioblastoma multiforme $(n=5)$ were included in the study. They were diagnosed after a precise clinical examination, laboratory and image analysis. Tissue samples taken intraoperatively were processed for routine pathohistological assessment. All patients signed an informed consent. They were regularly followed up after the surgical 
treatment in the Department of Neurosurgery. The study was approved by the Ethics Committee of Medical UniveristyPlovdiv.

Peripheral blood was collected preoperatively from all patients for immune status detection. No previous treatment with antitumoral agents or radiation was applied. Routine haematological and biochemical analyses were also performed.

Lymphocyte subsets were evaluated in whole blood by Cytomix FC500 flowcytometer (Beckman-Coulter Inc, USA). Two-color phenotypic characterizations of lymphocytes were performed using directly labeled FITC- and PE- monoclonal antibodies to: CD19, CD3, CD4, CD8, CD56,CD25, CD11b. All antibodies and reagents were obtained from Beckman-Coulter and staining procedures were performed following manifacturer's instructions.

Statistical analysis was completed by Statistica 6 . Mean value and standard deviation of populations studied were compared to age-matched reference values for each cell population.

\section{RESULTS}

The patients included in the investigation were operated and examined in a period of 6 months (November 2009 - April 2010). Three of the GBM patients and one with anaplastic astrocytoma grade III died from 0.5 to 3.5 months after diagnosis and surgery. None of them have had any radio- or chemotherapy.

No significant changes were detected in CD19+, $\mathrm{CD} 3+, \mathrm{CD} 3+\mathrm{CD} 4+, \mathrm{CD} 3+\mathrm{CD} 8+$ cell populations (Fig.1.). The CD4/CD8 ratio was not altered in most of the patients except in the one with the shortest survival time $(0.5$ months $)$. He had a diminished CD4/CD8 ratio of 0.6 compared to the reference range $(0.9-2.0)$. Only in this patient a significantly increased number (31.8) of NKT cells (CD3+/CD56+) was noted compared to the mean value (8.5) for the target group.

No obvious decrease in NK (CD3-CD56+) and NKT $(\mathrm{CD} 3+\mathrm{CD} 56+)$ cells was observed in most patients. Only in 2 cases of astrocytoma grade II reduced NK cell numbers were registered.

As a whole the total number of white blood cells for all patients was in the reference range. The differential white blood cell count in GBM demonstrated a significantly expressed lymphopaenia $($ mean $=12.4 \%$ ) with a relative peak in neutrophils (mean $=81.9 \%$ ) (Fig.2.). In the patient with the most rapid lethal outcome lymphocytes were only $2.7 \%$.

Interestingly, a reproducible phenomenon of increased $\mathrm{CD} 8+\mathrm{CD} 11 \mathrm{~b}+$ (mean value 22.4) and decreased CD8+CD11bcells (mean value 11.1) was found (Fig.3.). The four patients with lethal outcome showed high percentage of CD8+CD11b+ cells. Again the one with the shortest survival was presented with the highest CD8+CD11b+ values.

\section{DISCUSSION}

The results from the immunophenotyping of patients with malignant glioma revealed no changes in humoral immunity. CD19+ cells of patients were within the reference range values.

On the contrary, cellular immune response was significantly disturbed indicating increased suppressor cells $(\mathrm{CD} 8+\mathrm{CD} 11 \mathrm{~b}+)$ and decreased cytotoxic CD8+CD11b- cells. It was shown that certain T-cell abnormalities can be temporarily reversed following tumor resection and afterwards return with tumor recurrence indicating that the suppressive phenotype rather than an overall defect in cellular immunity matters (7). It is interesting thus to follow up prospectively the immune status of these patients.

The GBM-associated immune suppression can be explained on one hand with circulating $\mathrm{T}$ cells entering the tumor environment which can potentially respond specifically to antigens. On the other hand, most peripheral $\mathrm{T}$ cells are never closely exposed to tumor cells and glioblastoma patients are not globally immunocompromised (8). These observations prompt that the tumor itself might act as a driving force behind the generation of the immunosuppressive effect. The fact that in the patient with postoperative survival of 0.5 months the CD4/CD8 ratio dropped down and the number of NKT cells (CD3+/CD56+) was augmented suggests that the altered systemic immune response is essential for tumor progression. The four patients with lethal outcome showed high percentage of CD $8+C D 11 b+$ cells suggesting that the balance between $\mathrm{T}$ cells with suppressor and cytotoxic phenotypes is crucial for the survival of GBM patients.

Recent studies indicate that glioma cells express either surface markers or secreted factors that act as immunosuppressive molecules on local activated T-cells. The expression of FasL on glioma cells hypothesizes the potential killing of activated tumor-specific T cells by close contact with FasL+ tumor cells (9). A mutation in phosphatase and tensin homologue (PTEN) and increased expression of the costimulatory molecule B7-H1 have been detected in glioma cells (10). PTEN-deleted cells were less susceptible to the cytotoxic effect of $\mathrm{CD} 8+$ cells and thus contributed to the immunosuppressive effect in GBM.

The lymphopaenia observed in all GBM patients is another point in favor of the downregulated systemic immune response. Although glioma cells themselves could produce suppressive factors and thus inhibit locally activated $\mathrm{T}$ cells, they could not provide a satisfactory explanation for the systemic immune suppression observed in GBM patients.

Address for correspondence

Victoria Sarafian, MD, PhD, DMedSci

Department of Medical Biology, Medical University-Plovdiv 15a, V. Aprilov Blvd., 4000-Plovdiv, Bulgaria Tel. +359/32/602 531; 602 993; e-mail: sarafian@abv.bg 
There are several vague aspects of immune dysfunction in malignant glioma that have to be elucidated. The pre- and postoperative monitoring of GBM patients seems to be a good point in estimating the prognosis of these highly malignant tumors.

\section{CONCLUSION}

This pilot study reveals the presence of altered immune response in malignant gliomas and opens possibilities for prospective investigations concerning immune status and clinical outcome.

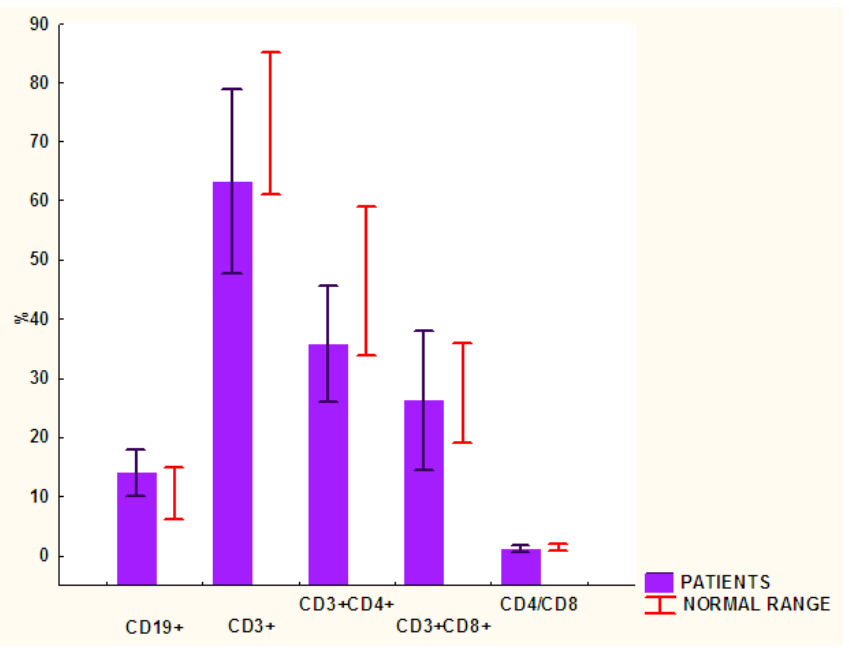

Figure 1. Expression of $\mathrm{CD} 19+, \mathrm{CD} 3+, \mathrm{CD} 3+\mathrm{CD} 4+$, $\mathrm{CD} 3+\mathrm{CD} 8+$ cell populations and $\mathrm{CD} 4 / \mathrm{CD} 8$ ratio in GBM.

Acknowledgement: The investigation is supported by grant No. 01/2009 from Medical University-Plovdiv. The authors thank Dr. H. Jelyazkov for his assistance in patients' management.

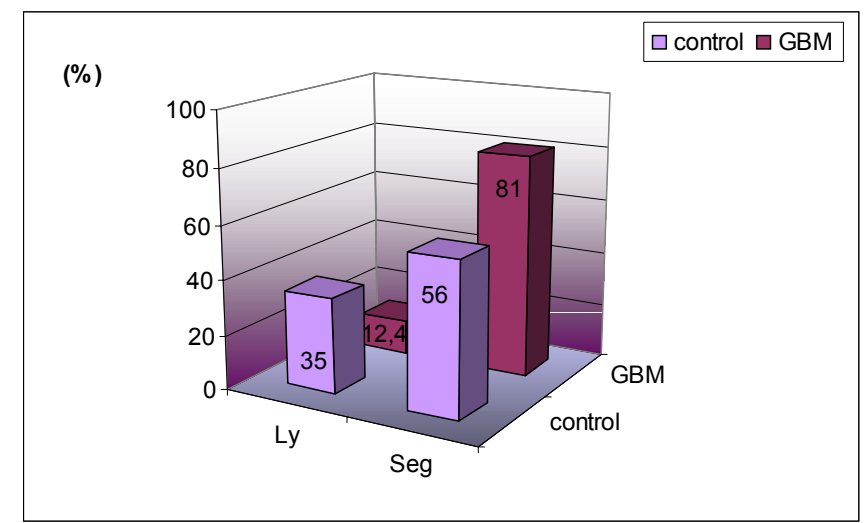

Figure 2. Changes in differential white blood cell count in GBM.

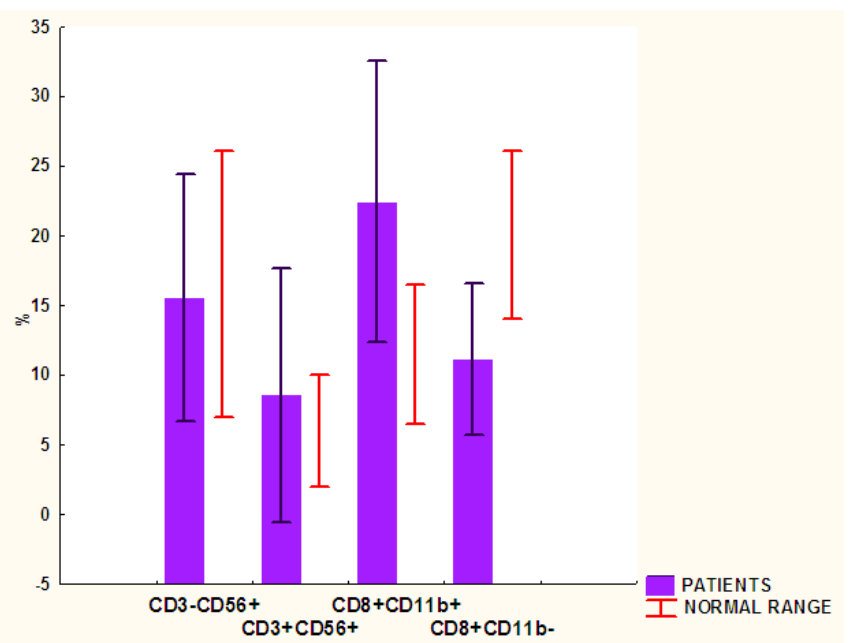

Figure 3. Altered expression of $\mathrm{CD} 8+\mathrm{CD} 11 \mathrm{~b}+$ and $\mathrm{CD} 8+\mathrm{CD} 11 \mathrm{~b}-$ molecules in GBM.

\section{REFERENCES:}

1. Sathornsumetee S, Rich JN. New treatment strategies for malignant gliomas. Expert Rev Anticancer Ther, 2006; 6 : 1087104.

2. Dix AR, Brooks WH, Roszman TL et al. Immune defects observed in patients with primary malignant brain tumors. J Neuroimmunol, 1999;100 : 216-232.

3. Gustafson MP, Lin Y, New KC et al. Systemic immune suppression in glioblastoma: the interplay between CD14 +HLADRlo/neg monocytes, tumor factors, and dexamethasone. Neuro Oncol, 2010 Feb 23.

4. Chiu TL, Lin SZ, Hsieh WH et al. AAV2-mediated interleukin-12 in the treatment of malignant brain tumors through activation of NK cells. Int J Oncol, 2009; 35: 1361-1367.

5. Castriconi R, Daga A, Dondero A et al. NK cells recognize and kill human glioblastoma cells with stem cell-like properties. J Immunol, 2009;182:35303539.

6. Wei J, Barr J, Kong LY et al. Glioblastoma cancer-initiating cells inhibit Tcell proliferation and effector responses by the signal transducers and activators of transcription 3 pathway. Mol Cancer Ther, 2010; 9 : 67-78.

7. Kempuraj D, Devi RS, Madhappan B et al. T lymphocyte subsets and immunoglobulins in intracranial tumor patients before and after treatment, and based on histological type of tumors. Int J Immunopathol Pharmacol, 2004; 17 : 57-64.

8. Waziri A. Glioblastoma-derived mechanisms of systemic immunosuppression. Neurosurg Clin N Am, 2010; 21: 31-42.

9. Ichinose M, Masuoka J, Shiraishi T et al. Fas ligand expression and depletion of T-cell infiltration in astrocytic tumors. Brain Tumor Pathol, 2001; 18 : 37-42.

10. Parsa AT, Waldron JS, Panner A et al. Loss of tumor suppressor PTEN function increases B7-H1 expression and immunoresistance in glioma. Nat Med, 2007; $13: 84-88$. 